\title{
FINITE RANK INTERMEDIATE HANKEL OPERATORS AND THE BIG HANKEL OPERATOR
}

\author{
TOMOKO OSAWA
}

Received 28 March 2006; Accepted 28 March 2006

Let $L_{a}^{2}$ be a Bergman space. We are interested in an intermediate Hankel operator $H_{\phi}^{M}$ from $L_{a}^{2}$ to a closed subspace $M$ of $L^{2}$ which is invariant under the multiplication by the coordinate function $z$. It is well known that there do not exist any nonzero finite rank big Hankel operators, but we are studying same types in case $H_{\phi}^{M}$ is close to big Hankel operator. As a result, we give a necessary and sufficient condition about $M$ that there does not exist a finite rank $H_{\phi}^{M}$ except $H_{\phi}^{M}=0$.

Copyright (c) 2006 Hindawi Publishing Corporation. All rights reserved.

Let $D$ be the open unit disc in $\mathbb{C}$ and let $d A$ be the normalized area measure on $D$. When $d A=r d r d \theta / \pi$, let $L^{2}=L^{2}(D, d A)$ be the Lebesgue space on the open unit disc $D$ and let $L_{a}^{2}=L^{2} \cap \operatorname{Hol}(D)$ be a Bergman space on $D$. When $M$ is the closed subspace of $L^{2}$ and $z M \subseteq M, M$ is called an invariant subspace. Suppose that $z L_{a}^{2} \subseteq M$. $P^{M}$ denotes the orthogonal projection from $L^{2}$ onto $M$. For $\phi$ in $L^{\infty}$, the intermediate Hankel operator $H_{\phi}^{M}$ is defined by

$$
H_{\phi}^{M} f=\left(I-P^{M}\right)(\phi f) \quad\left(f \in L_{a}^{2}\right)
$$

When $M=L_{a}^{2}, H_{\phi}^{M}$ is called a big Hankel operator and when $M=\left(\bar{z} \overline{L_{a}^{2}}\right)^{\perp}, H_{\phi}^{M}$ is called small Hankel operator. $L^{2}$ has the following orthogonal decomposition:

$$
L^{2}=\sum_{j=-\infty}^{\infty} \oplus \mathscr{L}^{2} e^{i j \theta},
$$

where $\mathscr{L}^{p}=L^{p}([0,1), 2 r d r)$ for $1 \leq p \leq \infty$. Set

$$
\mathbf{H}^{2}=\sum_{j=0}^{\infty} \oplus \mathscr{L}^{2} e^{i j \theta},
$$

then $L_{a}^{2} \subset \mathbf{H}^{2} \subset\left(\bar{z} \overline{L_{a}^{2}}\right)^{\perp}$. 
2 Finite rank intermediate Hankel operators

For an invariant subspace $M$, set

$$
M_{j}=\left\{f_{j} \in \mathscr{L}^{2} ; f \in M, f(z)=\sum_{j=-\infty}^{\infty} f_{j}(r) e^{i j \theta}\right\} .
$$

We call $\left\{M_{j}\right\}_{j=-\infty}^{\infty}$ the Fourier coefficients of $M$ and then $r M_{j} \subseteq M_{j+1}$. If $M_{j} e^{i j \theta}$ belongs to $M$ for any $j$, then $M$ has the following decomposition:

$$
M=\sum_{j=-\infty}^{\infty} \oplus M_{j} e^{i j \theta}
$$

When $M \subseteq \mathbf{H}^{2}, H_{\phi}^{M}$ is close to big Hankel operator. In this case, we give a necessary and sufficient condition about $M$ that there does not exist a finite rank $H_{\phi}^{M}$ except $H_{\phi}^{M}=0$.

The following lemma is proved in previous paper [1].

Lemma 1. Suppose $M$ is an invariant subspace which contains $z L_{a}^{2}$, and $\phi$ is a function in $L^{\infty} . H_{\phi}^{M}$ is of finite rank $\leq \ell$ if and only if $\phi$ belongs to $M^{\infty, \ell}$, where

$$
M^{\infty, \ell}=\left\{\phi \in L^{\infty} ; b \phi(z) \in M, b(z)=\sum_{j=0}^{\ell} b_{j} z^{j} \text { and } b_{j} \in \mathbb{C}\right\} .
$$

Note that we have proved in the previous paper [1, Theorem 5.4(1)] only when $k=0$. We improve [1, Theorem 5.4]. That is the following theorem.

Theorem 2. Suppose $M$ is an invariant subspace between $z L_{a}^{2}$ and $e^{-i k \theta} \mathbf{H}^{2}$ where $k \geq 0$, and $\phi=\sum_{j=1+k}^{\infty} \phi_{-j}(r) e^{-i j \theta}$ is a function in $L^{\infty}$. Then there does not exist any finite rank $H_{\phi}^{M}$ except for $H_{\phi}^{M}=0$ if and only if $M_{-(k-j)} \cap r^{j+1} \mathscr{L}^{\infty}=\{0\}$ for any $j \geq 0$.

Proof.

$$
\int \sum_{j=1+k}^{\infty} \phi_{-j}(r) e^{-i(j-m) \theta} \frac{d \theta}{2 \pi}=\phi_{-m}(r) \quad(1+k \leq m \leq \infty),
$$

and so

$$
\begin{aligned}
\left|\phi_{-m}(r)\right| & \leq \int\left|\sum_{j=1+k}^{\infty} \phi_{-j}(r) e^{-i(j-m) \theta}\right| \frac{d \theta}{2 \pi} \\
& =\int\left|e^{-i m \theta}\right|\left|\sum_{j=1+k}^{\infty} \phi_{-j}(r) e^{-i(j-m) \theta}\right| \frac{d \theta}{2 \pi}=\int|\phi| \frac{d \theta}{2 \pi}<\infty .
\end{aligned}
$$

Hence $\phi_{-m}(r) \in \mathscr{L}^{\infty}$ for $1+k \leq m \leq \infty$. If $r\left(H_{\phi}^{M}\right) \leq \ell(<\infty)$ by Lemma 1 then there exist complex numbers $b_{0}, \ldots, b_{\ell}$ such that $b_{\ell}=1, b=\sum_{j=0}^{\ell} b_{j} z^{j}$ :

$$
b \phi=\sum_{n=-\infty}^{\ell-(1+k)}\left(\sum_{m=0}^{\ell} b_{m} r^{m} \phi_{n-m}(r)\right) e^{i n \theta} \in M .
$$


Since $M \subseteq e^{-i k \theta} \mathbf{H}^{2}$,

$$
b \phi=\sum_{n=-k}^{\ell-(1+k)}\left(\sum_{m=0}^{\ell} b_{m} r^{m} \phi_{n-m}(r)\right) e^{i n \theta} \in M \quad(-(\ell+k) \leq n-m \leq \ell-(1+k)) .
$$

Since $M=\sum_{j=-\infty}^{\infty} \oplus M_{j} e^{i j \theta}$, by $(10)$,

$$
\sum_{m=0}^{\ell} b_{m} r^{m} \phi_{n-m}(r) \in M_{n} \quad(-k \leq n \leq \ell-(1+k)) .
$$

As $n=\ell-(1+k)$,

$$
\sum_{m=0}^{\ell} b_{m} r^{m} \phi_{n-m}(r)=r^{\ell} \phi_{-(1+k)}(r) \in M_{\ell-(1+k)}
$$

If $\phi_{-(1+k)}(r) \neq 0$, then $M_{\ell-(1+k)} \cap r^{\ell} \mathscr{L}^{\infty} \neq\{0\}$. So we assume $\phi_{-(1+k)}(r)=0$. As $n=\ell-$ $(2+k)$,

$$
\sum_{m=0}^{\ell} b_{m} r^{m} \phi_{n-m}(r)=r^{\ell} \phi_{-(2+k)}(r) \in M_{\ell-(2+k)}
$$

If $\phi_{-(2+k)}(r) \neq 0$, then $M_{\ell-(2+k)} \cap r^{\ell} \mathscr{L}^{\infty} \neq\{0\}$ and so $M_{\ell-(2+k)} \cap r^{\ell-1} \mathscr{L}^{\infty} \neq\{0\}$. So we assume $\phi_{-(2+k)}(r)=0$. Repeating the same way from $n=\ell-(3+k)$ to $n=\ell-(\ell-1+k)$, we can get $\phi_{-(3+k)}(r)=\cdots=\phi_{-(\ell-1+k)}(r)=0$. As $n=-k$,

$$
\sum_{m=0}^{\ell} b_{m} r^{m} \phi_{n-m}(r)=r^{\ell} \phi_{-(\ell+k)}(r) \in M_{-k}
$$

If $\phi_{-(\ell+k)}(r) \neq 0$, then $M_{-k} \cap r^{\ell} \mathscr{L}^{\infty} \neq\{0\}$ and so $M_{-k} \cap r \mathscr{L}^{\infty} \neq\{0\}$. If $\phi_{-(\ell+k)}(r)=0$, then $\phi_{-(1+k)}(r)=\phi_{-(2+k)}(r)=\cdots=\phi_{-(\ell+k)}(r)=0$ and $\phi=0$ by (10). This result contradicts $H_{\phi}^{M} \neq 0$, and so $M_{j-k} \cap r^{j+1} \mathscr{L}^{\infty} \neq\{0\}$ for $j \geq 0$.

If $r^{j+1} f \in M_{j-k} \cap r^{j+1} \mathscr{L}^{\infty}\left(f \in \mathscr{L}^{\infty}\right)$, then put $\phi=f e^{-i(k+1) \theta} \in L^{\infty}$. If $f \neq 0$, then $\phi \notin$ $M$ and

$$
z^{j+1} \phi=r^{j+1} f e^{i(j-k) \theta} \in M_{j-k} e^{i(j-k) \theta} .
$$

Since $M=\sum_{j=-\infty}^{\infty} \oplus M_{j} e^{i j \theta}, M_{j-k} e^{i(j-k) \theta} \subseteq M$ and so $z^{j+1} \phi \in M$. Lemma 1 gives a contradiction.

\section{References}

[1] T. Nakazi and T. Osawa, Finite-rank intermediate Hankel operators on the Bergman space, International Journal of Mathematics and Mathematical Sciences 25 (2001), no. 1, 19-31.

Tomoko Osawa: Mathematical and Scientific Subjects, Asahikawa National College of Technology, Asahikawa 071-8142, Japan

E-mail address: ohsawa@asahikawa-nct.ac.jp 


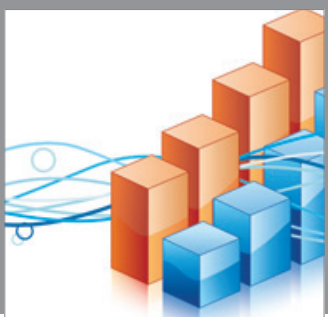

Advances in

Operations Research

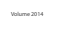

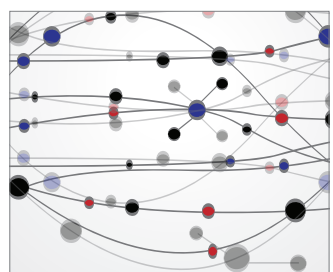

\section{The Scientific} World Journal
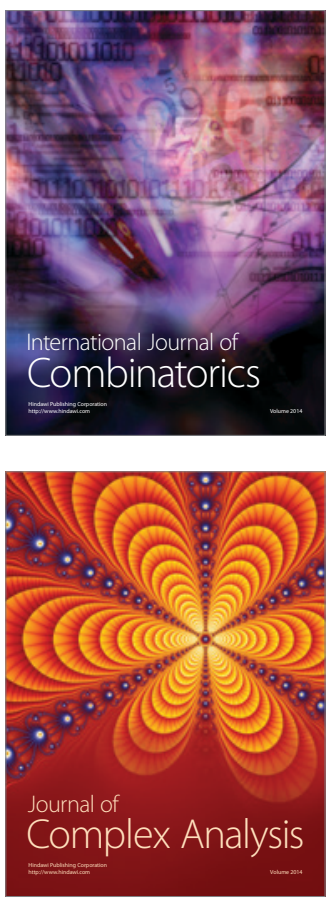

International Journal of

Mathematics and

Mathematical

Sciences
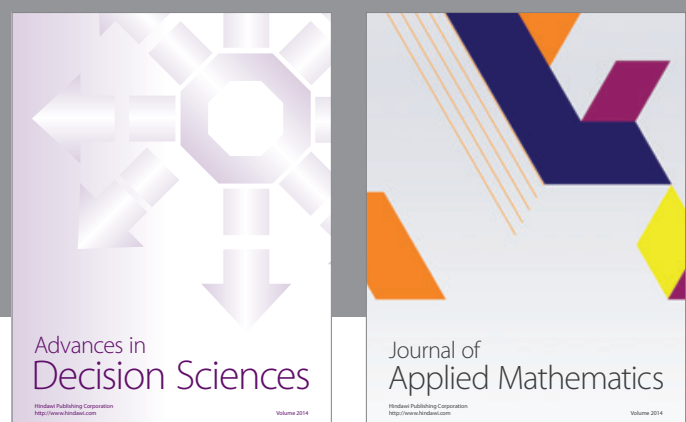

Journal of

Applied Mathematics
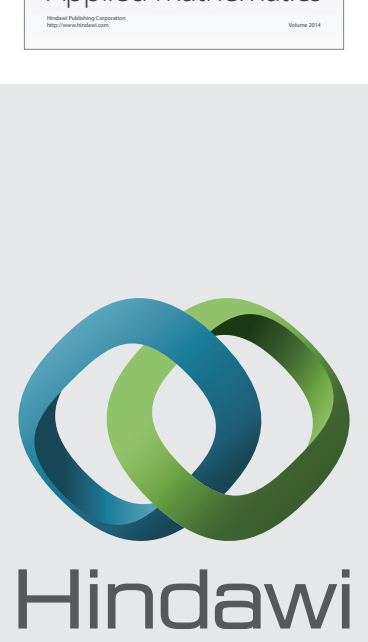

Submit your manuscripts at http://www.hindawi.com
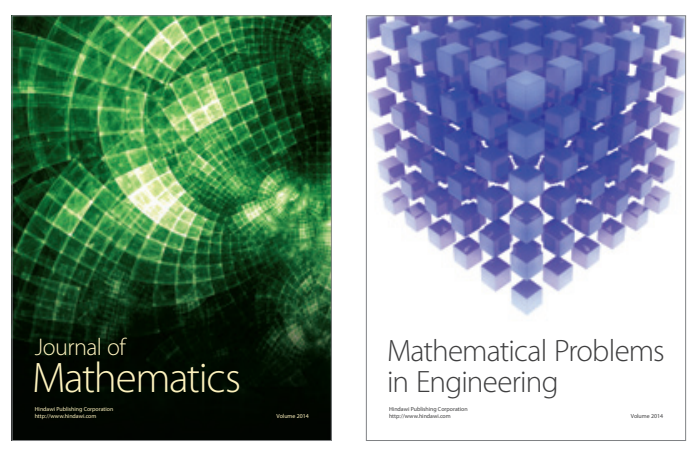

Mathematical Problems in Engineering
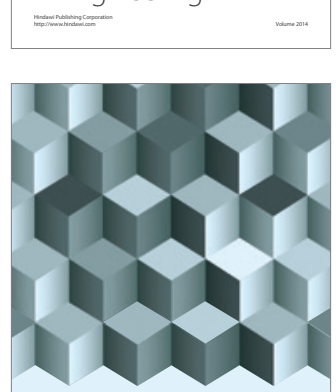

Journal of

Function Spaces
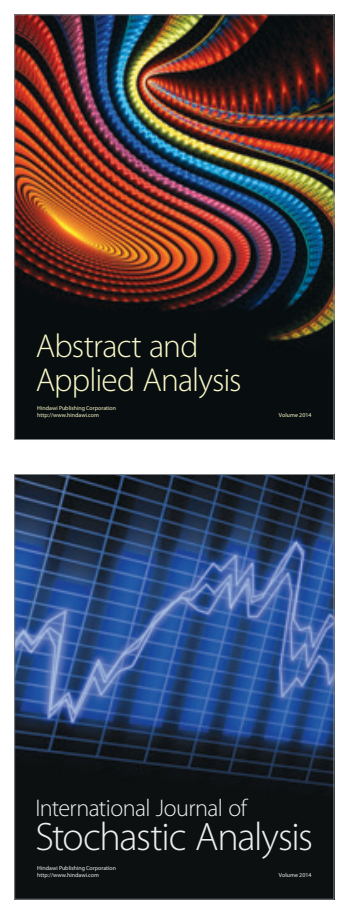

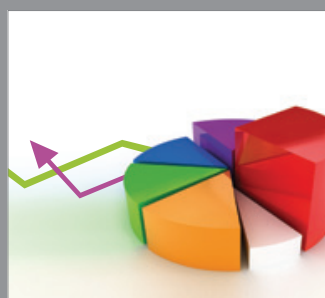

ournal of

Probability and Statistics

Promensencen
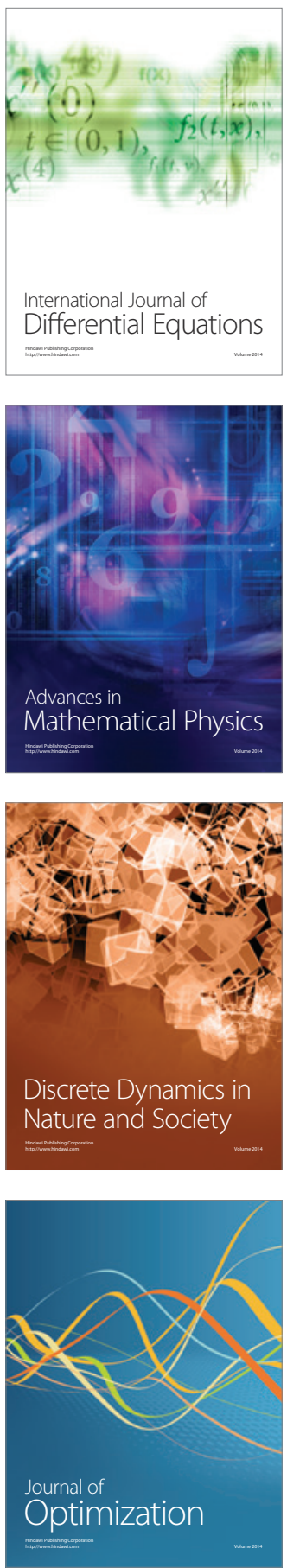